\title{
PAIN MANAGEMENT AND SCIENTIFIC ACUPUNCTURE
}

\author{
Kumio YAMASHITA. M. D. \\ Tokai University Oiso Hospital \\ Professor of Oriental Medicine
}

Although my presentation today, concerns Pain Management and Scientific Acupuncture, I would like to give you a brief outline on the history of Acupuncture in Japan.

\section{I . A Brief History of Acupuncture in Japan}

It is generally believed that acupuncture was brought to Japan from China by Chi So (Zhi Cong) (智聡). However, some Japanese historians believe this was done by Jo Fuku (Xu Fu) (徐福) in 265 A. D..

In 608 two Japanese monks, Enichi（恵日） and Fukuin（福因）, studied acupuncture in China. In 984, the noted physician, Tanba Yasuyori (丹波康頼), published "Ishinpo" (医心 方), the oldest medical textbook in Japan in which the practice of acupuncture was described. The Edo period $(1603$ - 1867) was the zenith for acupuncture in Japan and most of the present acupuncture technique was developed during this period.

After the Meiji Restoration in 1868, the Japanese government established a medical law for Western medicine which effectively omitted Ancient Traditional Chinese Medicine, referred to as Kampo in Japanese and which included acupuncture, from formal medical school education. Acupuncture, moxibustion, oriental massage, and other techniques continued to be practiced with the support of many patients, and were officially recognized under Japanese law in 1947.

It is interesting, however, that some of the medical doctors educated in Western medicine maintained a deep interest in Kampo. Among them was Yoshio Nakatani who studied acupuncture electrophysiologically and discovered that most of the meridian points of the traditional acupuncture theory corresponded to points which have lower electrical resistance than the normal body surface. These meridian points are called "tsubo" in Japanese. Nakatani named these points "Ryodoten" which means electroconductive points, and the systematic functional group of Ryodoten which form the shape of a band, he called "Ryodoraku".

It is the consensus of many medical researchers today that the Ryodoraku corresponds to the meridian pathway of Traditional acupuncture theory. By using this Ryodoraku therapy for pain management, we have successfully treated many cases. 
Turning to the Japanese history of acupuncture medicine, I will point out some interesting facts. According to the Taiho statute in $701 \mathrm{~A}$. D., the training course for acupuncture was described as 7 years. This is very interesting as this nearly compares with the length of present Western medical education which is 6 years.

Isai Misono devised an acupuncture method using a rather thick and dull needle and tapped it in with the inertial strength of a wooden hammer. Once he treated a weakened and nearly withered peony in the emperor's garden and revived it with acupuncture. The emperor was so pleased that he awarded him the honorable family name of Misono, which means the palace garden.

Waichi Sugiyama one of the well-known acupuncturists in the Edo period, (ruled by the Tokugawa), who because of blindness was'nt able to learn acupuncture technique very well, one day visited the Enoshima Cave Shrine to pray for help to enable him to do his work better. He promised the god to continue his prayer for 100 days. On the last day of his promise, there was no sign of help from the god and he fell down and wept. Just then, a sharp needle wrapped in a wide leaf pricked his bottom; at that time it suddenly occured to him that acupuncture could be done more easily by using a tube to pass the needle through. In spite of his handicap of blindness he became an excellent acupuncturist by using tubes. The development of the needle tube by a blind person became the basis for later developments such as the Showa needle tube, which is more commonly known as the Ryodoraku Autonomic Nervous System Regulating Needle.

Since 1971, when acupuncture analgesia was broadcasted to many countries from the People's Republic of China by reporters of the United States who accompanied President Nixon, acupuncture and Chinese herbal medicine were praised by journalism throughout the world.

However, 4 years prior to the lst Chinese acupuncture analgesia report in China, Kobei Akabane (a renowned acupuncturist) and Hideo Nishiyama (a gynaecologist) used acupuncture analgesia for child delivery.

In 1969, Michio Tani and Yoshio Manaka used it for 3 cases of appendectomy.

In 1950, Dr. Nakatani discovered Ryodo points while measuring the electroconductivity of a patient with very severe nephrtic oedema.

Dr. Nakatani noticed many electro-permeable points on the patient's skin and revealed that these points were not much different from those of other patients who suffered from the same nephritic oedema.

He also noticed that these electro-permeable points, or Ryodo points as he called them, were very similar in location with the classic meridian points indicated by Traditional Acupuncture Theory.

He also noticed that Ryodo points connected a line of electro-permeable points similar to, but not exactly like the classic meridian points. That is to say, there are some important differences.

"Ryodo" means high electrical conductivity and "Raku" means a connected line of points, thus Ryodoraku signifies a line of high electrical conductivity.

The meridian points and meridian lines were revealed clearly in the famous bronze figure which is said to have been made in 1443 A. D. during the Ming Dynasty. The phenomenon of 
the 12 meridians was discovered nearly 1500 years ago. Since this date no major changes or corrections have taken place.

These meridian lines are now studied as P. S. C. or P. S. M. (Propagated Sensation along Channels or Meridians), not only in China, but also worldwide including Japan.

Whereas the use of acupuncture analgesia has declined, the number of hospitals where acupuncture treatment is being used has increased gradually since 1975. Even though acupuncture analgesia for surgery is gradually becoming less widely used in Japan, acupuncture treatment derived from acupuncture analgesia is becoming more popular in the big hospitals. Now nearly $80 \%$ of the big hospitals are using acupuncture medicine, especially in their pain clinics; about $2 \%$ of national Japanese medical care consists of acupuncture treatment.

Areas which acupuncturists are specializing in are summarized as follows :

@ The Four Examinations (Looking, Listening and Smelling, Asking and Touching) YinYang Theory, and Five Phase Theory. $\quad 40 \%$

(a) Modern Empirical Acupuncture (Combination of Traditional and Modern

Scientific Approaches with a strong background in the health sciences, including Anatomy, Physiology, and Pathology) 25\%

(a) Ryodoraku Autonomic Nervous System Regulating Therapy 10\%

(a) Modern Chinese Acupuncture $10 \%$

@Combined or Others: $\quad 15 \%$

Note: Among Western medical doctors in Japan, Ryodoraku is the more popular method of treatment.

\section{Features of Ryodoraku}

a) General treatment

As you know, acupuncture treatment consists of three methods, 1 ) general treatment 2) local treatment and 3 ) specific treatment.

As for general treatment, usually 4 traditional methods of examination are used: they are Looking, Listening, Asking, and Touching (Palpation). These classic methods rely mainly on subjective evaluation, which makes it difficult to correlate and evaluate patient data.

In Ryodoraku therapy, an objective measuring method has been devised by using electropermeable points. The points are detected with an instrument named the Neurometer, which is equipped with a grip electrode for the indifferent electrode, electric battery, variable resistor, micro-meter, voltage-selector and measuring electrode. The patient grips the positive electrode in one hand, while the examiner runs a negative moist electrode, with a saline-soaked cotton ball fitted into the ebonite cap, along the patient's skin. Note the use of a moist electrode is unrelated to the function of sweat glands and hair follicles.

Before performing Ryodoraku measurement, the moist electrode and the grip electrode are connected and the current is abjusted to 200 micro amperes at 12 volts.

In order to determine the extent of abnormalities of the Ryodoraku, a specially designed Ryodoraku chart is used. The value of electric current is measured at representative measuring points on each Ryodoraku, and the value of these points is plotted on the chart with a short horizontal line. 
Ryodoraku representative measuring points on the hand are arranged along the wrist and nearly coincide with the Source Points of the respective meridians. Differences between Representative Measuring Points and Meridian Points are seen on the Small Intestine and Large Intestine meridians.

For example,

(Small Intestine Representative measuring point is $\mathrm{H}_{4} 5$ (SI 5 ) and the Source Point is $\mathrm{H}_{4} 4$ (SI 4 ))

(Large Intestine Representative measuring Point is $\mathrm{H}_{6} 5$ (LI 5 ) and the Source Point is $\mathrm{H}_{6} 4$ (LI 4))

Ryodoraku measuring points on the foot also nearly coincide with the Source Points, except for the Kidney and Urinary Bladder meridians.

For example,

(Kidney meridian Representative measuring point is $\mathrm{F}_{3} 6$ (KI 4 ) and the Source Point is $\mathrm{F}_{3} 3$ (KI 6) and Bladder meridian Representative point is $\mathrm{F}_{4} 3$ (BL 65) and the Source Point is $\mathrm{F}_{4} 4$ (BL 64)

In the Ryodoraku chart, when the measured values are approximately on a single line across the chart, the patient may be considered to be in good health.

However, when a discrepancy appears showing high and low values of a divergence of more than $1.4 \mathrm{~cm}$, this would indicate abnormalities. If this $1.4 \mathrm{~cm}$ band across the chart is taken as the patient's normal physiological range, when one of the Ryodoraku shows values higher (excitation) or lower (inhibition) than the physiological range, in each case the Ryodoraku characteristic symptoms will be present.

As for Ryodoraku treatment, higher Ryodoraku readings may be supressed by using Sedation points and lower Ryodoraku readings may be stimulated by using Tonification points.

I have devised a new Ryodoraku chart which has two more Tonification and Sedation points added to the original Ryodoraku chart. By using this new chart, doctors will find it easier to choose treatment points in their daily clinical work.

b) Local treatment

As for local treatment, Ryodoraku has a very useful method called "REPP" (Reactive Electro Permeable Point) therapy.

The meridian points are specific reactive points and are the treatment points on the meridian line.

When a moist electrode using 12 volts is applied to a patient's skin, comparatively clear electropermeable points are revealed. They are called reactive electropermeable points or Reactive Ryodo points.

The reactive electropermeable points, depending on the disease, are the loci where excitation of the sympathetic nerves on the body surface is heightened by reflexes. When appropriate stimuli are administered to such REPP, in almost all cases, the electric permeability is lowered and the complaints of the patients decrease or disappear completely.

This is understood as follows: Localized excitation of the sympathetic nerves occurs at the body surface due to organ disorder or between nearly normal or abnormal tissue. Thus the REPP are considered effective points of treatment for acupuncture, and hence this is referred 
to as REPP therapy, or localized sympathetic nervous regulatory therapy.

c) Specific treatment

Specific treatment means to use one or several acupoints which are useful for a particular disease or its symptoms. There are many such points written in the classics, as well as in recent acupuncture books.

In Ryodoraku Therapy, one doesn't hesitate to use these specific points if necessary. I would like to mention several specific points which are very useful in Ryodoraku therapy.

1) Nakatani's A and B Eye Points.

Eye point $A$ is located on the line from the outer edge of the eyelid to the upper root of the ear lobe in front of the hair line. Eye point B is located in front of point A. Needles may be inserted down behind the zygomatic bone from these two points. A and B are very effective points for eye diseases, including color blindness and pseudo myopia.

2) $\mathrm{H}_{5} 30$ ( $\left.\mathrm{TH} 21\right)$ for tinnitus.

Insert the needle right into the artery: micro-bleeding from this point may be very successful for tinnitus and dizziness.

3 ) New point Bi-sen (also called Jo-gei-ko) for rhinitis.

$\mathrm{Bi}$-sen is located beside the nasal bone and $1 \mathrm{~cm}$ below $\mathrm{F}_{4} 75$ (BL 1 ). For nasal stimulation, the needle may be inserted downward along the line of the nose from the point just below the socket while rubbing the bone. 5 times of light sparrow pecking technique is sufficient.

This treatment is indicated for allergic rhinitis and rhinitis hypertrophica. In addition, stimulation on REPP is necessary for Empyema Highmori.

4) Gonitis REPP therapy

Surrounding the patella, two upper and two lower REPP are found. Sometimes one more REPP is found. From the base of the patella, the needle should be inserted behind the cavity of the patella. For cases of severe pain, two acupuncture insertions may be added contralaterally along the patella.

After the needle is inserted, it is pushed through the skin and pecked, or sometimes electrically stimulated, except in cases of rheumatism.

As you can see, one also uses various points in Ryodoraku treatment that are not selected directly from the Ryodoraku Chart.

These are summarized as :

1) REPP for local therapy 2) Painful pressure sensitive points or tender points 3 )

Reactive motor points found in stiff muscles 4) Points near nerve trunks 5 ) Direct organ stimulation if necessary 6) Points surrounding inflammation loci, and 7) Traditional meridian points which are indicated according to classical theory.

d) Features of Ryodoraku

In conclusion, the features of Ryodoraku are as follows:

1) Scientific -..-Treatment points are measured electrically.

2) Objective -...Meridian phenomena are objectively evaluated on the chart.

3 ) Simple -...Easy to use in clinical practice.

4) Clean and Safe -... One needle per patient and no needle contamination.

5 ) Comprehensive -...Easy to understand for any doctors who are not familiar with 
Eastern medical methods of thinking.

We can say that these features just mentioned, abbreviated as SOSCC warrant the recent increase of Ryodoraku in the big hospitals which have over 500 beds. According to recent statistics over $80 \%$ of them use acupuncture including Ryodoraku.

\section{The Future of Ryodoraku}

a) A New Ryodoraku Chart

Although there are many areas in which Ryodoraku can be improved, it has many excellent features, including those I have referred to as SOSCC, which insure that Ryodoraku will earn its place as a model scientific method of treatment. Recently a newly improved neurometer has been devised, the characteristics of which are as follows:

1) Abnormal Ryodoraku and left-right differences will be clearly discriminated by a circular graph.

2) It can record old and new data automatically and simultaneously so that the information can be displayed on the same screen.

3 ) The Ryodoraku treatment points, that is the General Regulatory Treatment of the Total Ryodoraku-GRT, can be displayed on a model of the human figure by an automatic analysis system. When requested this system will also provide a description for herbal medicine.

b) Cancer Research

Dr. Kobayashi suggested the Ryodoraku abnormalities specific to cancer. The theory is as follows: The autonomic nervous system, which supports the involuntary activities of daily life, is controlled by the sympathetic and parasympathetic nerves.

Excessive tension of parasympathetic nerves, which are necessary for noctural functioning, is implicated it the manifestation of cancer. Also he noticed that cancer patients usually have excitation of $\mathrm{H}_{2}(\mathrm{HC}), \mathrm{H}_{3}(\mathrm{HT})$ and $\mathrm{H}_{5}(\mathrm{TH})$, and inhibition of $\mathrm{F}_{1}(\mathrm{SP}), \mathrm{F}_{3}(\mathrm{KI})$, and $\mathrm{F}_{5}(\mathrm{~GB})$ and also show significant differences in values on the left and right sides of the body. From these observations he devised a Cancer Check of the Related Meridians (CCM).

Thus, Ryodoraku is now being used for the diagnosis of cancer.

c) Health Examination or human "dock" and Ryodoraku

I had the experience of working in a clinic which also had a health check system. In this clinic I used Ryodoraku for the health check; it was very successful because I could give the patients a clear and logical explanation using the Ryodoraku Physiological chart to show correlations between Ryodoraku patterns and specific health problems. A large percentage of them have become our clinic patients and we are treating their various complaints.

\section{Meridian Points}

As for meridian points, they are usually defined as the dotted points along the meridian and are said to be the entrance and exit of vital energy, vital energy being referred to a ' $\mathrm{Ki}$ ' in Japanese. They are the reactive points of several diseases, and at the same time, they are useful and effective points for the treatment of these diseases.

Briefly the meridian point is the entrance of vital energy and an important point for the treatment of these diseases. 
In Ryodoraku therapy, meridian points may be the loci where the excitation of the sympathetic and sensory nerves is caused by some disease, and in this sense, every part of the whole body can be an acupuncture point.

The meridian phenomenon observes the function of the sympathetic nerses on the surface of body, while the Ryodoraku phenomenon effectively demonstrates the level of excitation along a line of points by measuring electrical skin resistance.

Here, Dr. Nakatani attached importance to the sympathetic nerve function concerning acupuncture and believed that many kinds of meridian phenomenon are revealed through the sympathetic nerve function.

No specific nerve endings have been discovered in the meridian points, although it is supposed that they contain specific receptors and consist of a balanced system for maintaining homeostasis of the human body. It is not sure whether these functional structures are projections of the central nervous system or if they are signs of some electromagnetic system. If the latter is true, meridian points will surely be given important attention in the future.

Here we will present our own definition.

The meridian phenomenon is a functional phenomenon demonstrating similar reactions to many kinds of phenomenon revealed in the atomic field. (electric wave, atmospheric pressure, circumstantial temperature, body temperature, and so on,) This shows up more clearly in an unhealthy body. The depth and size of these meridian points differ according to individual body types.

"What is a meridian?" is the question which is likely the oldest and yet the newest question of all. The doctors who have an interest in acupuncture medicine and who use it in their daily clinic work, or physiologists and anatomists who are studying the mechanism of acupuncture analgesia and morphology of skin must answer this question in the near future.

Recently, it has become understood that the meridian or channel pathway are likely to be PSC (Propagated Sensation along Channels).

PSC was formally reported in 1979 in the People's Republic of China.

These phenomena had already been observed in Japan in 1974. The left side of the figure (omitted) shows the route of PSC drawn on the skin at the time of inserting a needle in BL $65\left(\mathrm{~F}_{4} 3\right.$ ) (Shugu) along the Bladder Meridian, and the upper right figure (omitted) shows the route of PSC when the needle is inserted into HT 7 (HT 3) (Shenmen) along the Heart Meridian. It was reported in 1974 as the needle sensation.

PSC is a phenomenon which is seen only in about $10-20 \%$ of people, not in everyone. It has the characteristic of transmitting from the point being stimulated to upper and lower regions.

It's conductive velocity is far slower than the speed of nerve conductivity and blood flow, and travels $1 / 10$ the velocity of the autonomic nerve.

In the upper extremities PSC is more clearly revealed than in the lower extremities.

Aside from meridians, there are some other theories which attempt to explain the projection of central nerves.

1) Thalamic Neuron Theory (Lee)

2 ) Propagated sensation along meridians (PSC) or (PSM)

3 ) Skin diseases along the meridians

These opinions can be classified as so-called functional theory or meridian theory. 
However, my interest in the theory of the morphological existence of meridians was aroused by the fact that the electro-permeable points can be shown to exist on the skin of breast tissue specimens, on amputated legs, and even dead bodies, several hours after death.

Dr. Kurabayashi of Japan, revealed that nerve-endings exist in nearly $100 \%$ of the loci of electro-permeable points (REPP) of dogs, cats, rabbits, rats, mice, and also in $30-40 \%$ of the loci of non-electropermeable points.

Now we can say at least that acupoints have many components of nerves and vessels when compared with non-acupoint areas.

Now, we have also found some electro-permeable points in concave places where the epidermis is rather thin.

When enlarged, you can see that the cells of the basal membrane are arranged horizontally which may relate to the good electro-permeability of certain areas. Good electro-permeability also tends to appear in concavities, valley, or hollows along the meridian. We tried to count the so-called volumedensities of nerves, blood vessels, and other elements in the skin. This drawing shows the area of each element, such as nerve fibers, blood vessels, and an outline of the skin surface. Blue indicates hair follicles, red shows blood vessels, and yellow shows the nervous elements. This slide (omitted) is a specimen from the popular acupoint ST 36 (Zu-sanri).

The next slide (omitted) shows the control area, outside the acupuncture point.

This slide (table) (omitted) summarizes our calculations. You can see the volume density of the acupoint ST 36 ( $\mathrm{Zu}$-sanri) is 8.82 compared to the control which is 2.25 . This means that the volume of the vessels at an acupoint is nearly three times as much as that in a non -acupoint.

The volume density of nerves is 7.22 / (versus) 5.28. This means that the volume of nerves in an acupoint is about one and half times as much as the volume in a non-acupoint. It was found that acupuncture points demonstrated the largest surface densities among the nervous elements and blood vessels of the skin, when compared with the control samples.

The following is my personal speculation:

The meridian may be like a plaza and once something happenes in the body, many of the so-called autocoids, that is automatically moving substances, come together at this plaza to transmit various kinds of information rapidly to the whole body through meridian pathways.

We can summarize the many theories concerning the meridian as follows:

\section{Meridian Point}

I . From a Morphological Perspective

1) Existence of specific ducts and bodies (Bong-Hang theory)

2 ) Existence of specific arterio-venous anastomosis (Niboyet)

3 ) Existence of specific subcutaneous induration (Fujita and Kishi)

4 ) Dense distribution of muscle bundles (People's Republic of China)

5 ) Dense distribution of nerve and vessels (Yamashita)

6 ) Others; (Pacinian corpuscles, lymph vessels, connective tissue)

II. From a Functional Reactive Point Perspective

1) Viscero-Cutaneous reflex, Oppressive pain points, Referred pain points 
2 ) Electro-permeable points:

a) Dermo-electric points (Ishikawa)

b) Ryodo points (Nakatani)

3) Points which demonstrate differences in shin temperature and perspiration III. From an Effective Point Stimulation Perspective

1) Trigger Points (Melzack)

2) Needle Sensation Points

3) Points which easily influence the internal viscera

4 ) Densely distributed areas of polymodal receptors

5 ) Densely distributed areas of A-beta nerve fibers

\section{Conclusion}

Ryodoraku therapy is not only an acupuncture theory with electrical stimulation, but also an oriental medicine using many kinds of ideas and techniques of Western medicine.

It must be emphasized that this therapy may be used easily and widely in western style clinics and hospitals. In the future we predict it may be used for the prevention of sickness, cancer research, stress alleviation, and even for hospice.

\section{Here's a bit of my own philosophy:}

Western medicine may be likened to a father's love, Oriental medicine to a mother's love, not one school of medicine is complete in itself; the combination, a Western-Oriental medical approach, that is to say, Ryodoraku, is the ideal par excellence. 